$\mathbf{X}$.

\title{
Bot Diodors annalistische Quelle die Namen der ältesten Volkstribunen?
}

Die Erkenntnis, daß die älteren römischen Annalen bei der kurzen Berichterstattung über die altrepublikanische Geschichte die Namen der Konsuln meist $\mathrm{n} i \mathrm{cht}$ genannt haben, ist bekanntlich durch Nieses Marburger Programmabhandlungen de annalibus Romanis (I. 1886 und II. 1888) bedeutend gefördert worden. Auch ich hatte dieses Ergebnis mit um so größerer Genugtuung begrüßft ${ }^{1}$ ), als allein bei einer so gearteten Annalistik der älteren republikanischen Zeit die chronologischen Schwierigkeiten dieser Epoche eine genügende Erklärung finden konnten.

Es mag sein, daß Niese an einigen Stellen aus dem Schweigen der Ueberlieferung über die Namen der durch die spätere sagenhafte Tradition bekannt gewordenen Persönlichkeiten zu viel geschlossen hat. Jedenfalls ist unter dem Einfluf der späteren Annalistik der Laudationen und der Elogia vielfach erst das persönliche Element in die Spezialdarstellung hineingetragen worden.

Um so bedauerlicher ist es, daß̧ Niese bei einer für die römische Verfassungsgeschichte besonders bedeutsamen Stelle eine bedenkliche Inkonsequenz gezeigt hat, indem er gerade die bei Diodor XI, 68 erwähnten Namen der 283 gewählten Volkstribunen als eine der gesichertsten Angaben alter Annalistik hingestellt hat.

Diese Vermutung muß um so mehr beanstandet werden, als sie zu mehreren falschen Hypothesen Anlaß3 gegeben hat.

1) Vgl. römische Chronologie (1889) S. $438 \mathrm{f}$. 
Die lex Publilia bestimmte nach Livius (II, 56) ut plebei magistratus tributis comitiis fierent, und entsprechend berichtet II, 58: tum primum tributis comitiis creati tribuni sunt; numero etiam additos tres, perinde ac duo fuerint, Piso auctor est; nominat quoque tribunos Cn. Siccium L. Numitorium M. Duellium Sp. Icilium L. Mecilium.

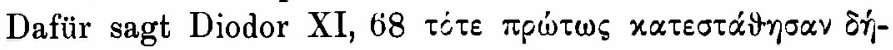

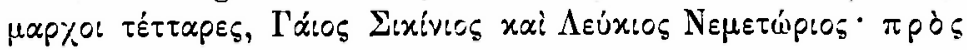

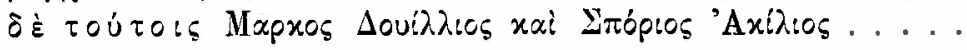

Niese hat hier, nach dem Vorgang von Eduard Meyer ${ }^{2}$ ), angenommen, daf Diodor, obgleich er sonst sehr sparsam in der Erwähnung von Namen ist, an dieser Stelle die Angaben der altern Annalen treuer wiedergegeben habe.

Gewiß ist es unzulässig, Diodor a priori nach Piso zu korrigieren ${ }^{2}$ ). Aber noch weniger ist es hier gestattet, Diodor allein zu folgen, Piso und alle anderen Annalenangaben wie Tuditanus und Cicero de republica zu vernachlässigen und olnehin nicht dasjenige festzuhalten, w orin D iod or und P is o übereinstimmen. Ob Pisos Vermutung von 5 Tribunen vernünftig, ob Diodors Annahme von 4 Tribunen richtiger ist, das kommt erst in $\mathrm{zweiter} \mathrm{L}$ in ie in Betracht. Wichtiger zu ergründen ist entschieden, was Diodor und den übrigen alten Annalisten gemeinsam ist. Denn erst dann kann der eventuelle Wert der eigenartigen Ueberlieferung bei Diodor festgestellt werden.

Schon Niese I, 11-13 war es nicht entgangen, dak Pisos und Diodors Bericht nicht nur die gleichen Tribunennamen böten, sondern auch die gleiche Reihenfolge beobachtet hätten. Auch sonst stim m e n die Berichte, abgesehen von der Variante in der Zahl (4 bezw. 5) formell so ü bere i n, dak sie mit Notwendigkeit auf einen g e me in s a m e n älteren Quellbericht zurückgeführt werden müssen. Derselbe würde etwa so gelautet haben:

tum primum tributis comitiis creati tribuni sunt $(\mathrm{Cn}$. Siccius L. Numitorius); numero

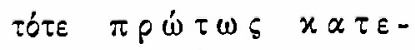
$\sigma \tau \alpha \vartheta \eta \sigma \alpha \nu \delta \dot{\eta} \mu \alpha \rho \chi \circ \imath \ldots$

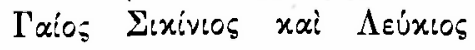

$\left.{ }^{2}\right)$ Eduard Meyer, Rhein. Museum 37, S. 617. 


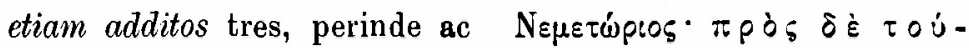

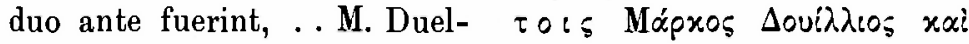

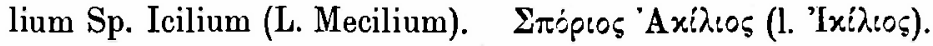

Mit Recht legte sich hier schon Niese die Frage vor

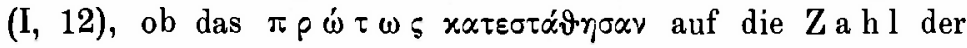
Tribunen gehe, oder vielmehr auf den Tribunat überha u t. Niese verwarf mit Grund die erste Eventualität; denn mag auch Diodor auf die Zahl 4 ein besonderes Gewicht gelegt haben, die ubereinstimmende Kombination der Worte

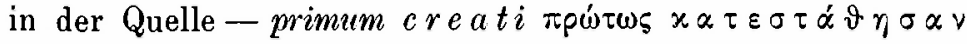
- legt das Hauptgewicht auf die Art der Erwählung, nicht auf die $\mathrm{Z}$ a hl.

Aber auch die zweite Möglichkeit kann nicht das Richtige treffen, denn nach Livius und nach Piso haben vor 283 bereits Tribunen fungiert. Ja, alle Quellen, die Cicero bekannt waren, - man denke namentlich an Tuditanus, Polybius, Piso - setzen die friihere Existenz des Tribunats voraus!

Es bleibt also dabei, daßs auch Diodor in seiner Quelle besonders hervorgehoben fand, daßi $\mathrm{damals} z \mathrm{u}$ erst eine

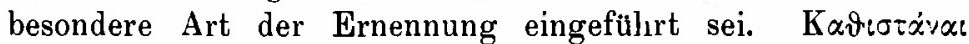
wird ja wie "creare" beinah technisch für die Erwählung der Beamten durch das Volk gebraucht. Auch bei Livius ist das eigenartige Neue die $\mathrm{W}$ a hl art durch die comitia tributa. Wie die Tribunenernennung früher erfolgt ist, darüber fehlte die Ueberlieferung. Das wird indirekt auch dadurch bezeugt, daßs manche sie in plebejischen Kurien oder Zenturien wählen ließen, und die Zahl der ersten Tribunen bestritten war ${ }^{3}$ ).

Aber noch mehr: die Quelle Diodors wie die Pisos gab ausdrücklich an, daß $\mathrm{dam}$ als ein Uebergang von der Zweizahl zu einer Mehrzahl stattgef un den habe.

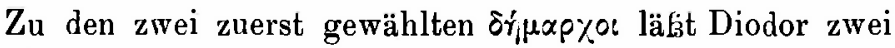
andere hinzugefügt werden. Unter keinen Umständen darf also aus Diodor die Folgerung gezogen werden, dak seine Quelle die Existenz der Volkstribunen vor 283 geleugnet habe. Auch ist eine solche bei den Berichten von Cicero de repu-

s) Vgl. Rom. Forsch. I, 185 und Soltau, die Anfänge der röm. Geschichtschreibung S. $251 \mathrm{f}$. 
blica, der nach Polybius und Piso schrieb, bei der übereinstimmenden Annahme aller übrigen Quellen über Zeit und Ort der 1. secessio ${ }^{4}$ ) und über die lex sacrata in monte sacro absolut unhaltbar ${ }^{5}$ ).

Man mag im weitern gern anerkennen, daß Niese vielen Scharfsinn angewandt hat, um den Nachweis zu erbringen, daß3 die 4 von Diodor genannten Tribunennamen, die ja Piso in der gleichen Reihenfolge neben einem 5. herzählt, einer relativ alten annalistischen Quelle angehört haben und vielleicht den Vorzug vor der Fünfzahl bei Piso verdienen. Was aber ist damit viel gewonnen?

Daßs die ältesten Annalen, welchen Diodor folgt, ohne Angabe von derartigen Personalien berichtet haben, ist gerade das Hauptergebnis von Nieses Untersuchungen gewesen, und er hätte es also vermeiden sollen, dieses Resultat wieder durch die Annahme, daß Diodors annalist is che Quelle derartige Einzelheiten gebracht habe, aufzuheben!

Aber die Quellenfrage bei Diodor ist über Ed. Meyers und Nieses Forschungen hinaus neuerdings vielfach noch weiter gefördert worden ${ }^{6}$ ). Am wichtigsten ist jedenfalls das Resultat, das Sigwart "römische Fasten und Annalen bei Diodor" gewonnen hat. Nach ihm finden sich im Diodor Auszüge aus einem lateinisch geschriebenen alten Annalisten mit den Angaben eines ebenfalls älteren griechischen Berichterstatters, dem auch die Fasten angehören, zusammengestellt.

Die sprachlichen Kriterien, welche bisher für eine doppelte Quelle der Fasten vorgebracht waren, sind nach Sigwart S. 5 f. mit Recht nicht als beweiskräftig anzusehen. Umsomehr aber ist festzuhalten, daß die $\mathrm{griech}$ is chen Fasten und manche Sonderberichte eines griechis ch schreibend e n Autors (vielleicht Kastor) getrennt und neben den Exzerpten aus einem latein is ch schreibenden Anna$\mathrm{l}$ is te $\mathrm{n}$ einhergehen ${ }^{7}$ ).

4) Die gute Ueberlieferung schied sehr wohl zwischen der 1. und 2. secessio, und wenn ausmalende Annalenberichte hier kleinere Züge von der einen in die andere hinübergenommen haben, so hat das keinen Quellenwert. Vgl. Soltau, die Anfünge der röm. Geschichtschreibung S. 166-168. S. auch Philol. (1897) 56, 118 f.

5) Man bedenke dabei, dafs das Wissen des Polybius wie des Piso auf dem alten Stadtbuch (der tabula, quam pontifex maximus proponebat domi) beruhte, wie Kornemann Klio $1911 \mathrm{~S}$. $245 \mathrm{f}$. gezeigt hat. Vgl. ferner Philol. (1896) 55, $269 \mathrm{f}$.

6) Klio 1905 , VI $269 \mathrm{f}$.

7) Es ist bedauerlich, daf Sigwart doch wieder meint, dafs beide Bestandteile schon in der Quelle Diodors kombiniert vorgelegen hätten. Damit hebt er seine eigene Beweisftuhrung wieder auf, welche auf der Tatsache einer verschiedenartigen Terminologie, je nachdem Diodor einer lateinischen oder einer griechischen Quelle gefolgt ist, beruht. 
Wenn dieses aber richtig ist, so können die Namen der Tribunen bei Diodor XI, 68 nicht aus der alten annalistischen Quelle stammen. Schon der Name $\Delta \circ v^{\prime}\left(\lambda \lambda \cos ^{8}\right)$ verrät die griechisch schreibende Fastenquelle. Entscheidend aber ist, dak, wie gesagt, die annalistische Quelle Diodors $n$ i e Namen der für das Jahr fungierenden Oberbeamten nennt, sondern sie nur dann erwähnt, wenn sie in die Handlung, in die Kriegführung eingreifen.

Welcher Herkunft aber sie auch sein mögen, und stammten sie selbst aus dem pontifikalen Kodex, dessen Existenz Kornemann so gut nachgewiesen hat ${ }^{9}$ ): das was bisher aus ihnen deduziert ist, ist unrichtig. Weder können sie wahrscheinlich machen, daß diese Tribunen di e er ste $n$ gewesen sind, noch daß die $\mathrm{Vi}$ e rzahl von irgend jemand als die ursprüngliche Zahl des Tribunenkollegiums angenommen worden ist. Alte Listen der Volkstribunen, das wird allgemein zugestanden ${ }^{10}$ ), sind nicht gefïhrt worden. Diese Namen sind also im besten Fall spätere Rekonstruktion, die vielleicht etwas äl t er ist, als das, was sonst über die 1. und 2. secessio plebis berichtet worden ist, ob aber besser?

Schon formell schließst sich die Notiz Diodors den Angaben d e s Chronographen an, dem Diodor seine Fasten verdankt, der bei den tribuni militum consulari potestate stets eine Summe gab, die noch dazu keineswegs immer zu den von Diodor gebotenen Namen stimmite ${ }^{11}$ ). - Nie hätte man endlich die Vierzahl der Tribunen mit der Zahl der städtischen Tribus in ein Verhältnis bringen sollen. Die Volkstribunen haben stets die Gesamtheit der Plebs vertreten, niemals die hauptstädtische Plebs allein, und noch weniger haben sie irgendwelche Beziehung zu einer einzelnen Tribus gehabt. Neben der Anfangszahl von 2 Volkstribunen und den späteren zehn ist alles das späte Kombination, was über das Verhältnis der Tribunen zu Tribus und Klassen vorgebracht ist. Jede alte Ueberlieferung fehlte hierüber und sollte also auch nicht durch moderne Einfälle ersetzt werden ${ }^{12}$ ). Zabern.

W. Soltau.

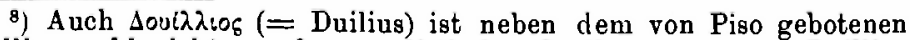
Duellius wohl nicht aus der annalistischen Quelle genommen. Duillius haben die Konsular- und Triumphalfasten, Duellius war sonst üblich Cic. Orat. 45. vgl. Pauly unter Duilii.

$\left.{ }^{9}\right)$ Klio XI, 253 f. $342 . \quad 10$ ) Auch von Niese I, 8 f.

11) Vgl. meine römische Chronologie S. 346.

12) Damit fällt das Kartenhaus zusammen, das K. J. Neumann (Römische Staatsaltertümer $\$$. 375 f.) auf der falschen Prämisse, daß 283 das Tribunat geschaffen sei, errichtet hat. Auch Eduard Meyer, Kleine Schriften S. $371 \mathrm{Anm} .1$ hält diesen Ansatz jetzt nicht mehr fest. 